Communication, technologies et développement

$6 \mid 2018$

Technologies mobiles, innovation et développement

\title{
Herramienta tecnológica de estimulación temprana para familias costarricenses
}

Instrument technologique de stimulation précoce pour des familles

costariciennes

Technological tool of early stimulation for costa rican families

Linda Madriz, Lorena Soto, Lennin Collado y Alex Segura

\section{OpenEdition}

Journals

Edición electrónica

URL: http://journals.openedition.org/ctd/619

DOI: $10.4000 /$ ctd. 619

ISSN: 2491-1437

Editor

Chaire Unesco Pratiques émergentes en technologies et communication pour le développement

Referencia electrónica

Linda Madriz, Lorena Soto, Lennin Collado y Alex Segura, « Herramienta tecnológica de estimulación temprana para familias costarricenses », Communication, technologies et développement [En línea], 6 | 2018, Publicado el 18 diciembre 2018, consultado el 19 septiembre 2019. URL : http:// journals.openedition.org/ctd/619; DOI : 10.4000/ctd.619

Este documento fue generado automáticamente el 19 septiembre 2019.

Communication, technologies et développement 


\title{
Herramienta tecnológica de estimulación temprana para familias costarricenses
}

\author{
Instrument technologique de stimulation précoce pour des familles \\ costariciennes \\ Technological tool of early stimulation for costa rican families
}

Linda Madriz, Lorena Soto, Lennin Collado y Alex Segura

1 El siguiente trabajo tiene como propósito presentar las acciones tomadas para la validación de una serie de escalas del desarrollo integral para la niñez, con edades comprendidas entre un mes hasta veinticuatro meses de vida (un total de diez) y que forman parte de la investigación: Detección, estimulación y atención temprana para la prevención de la discapacidad en niños y niñas costarricenses con una condición de riesgo biológico y/o social: Herramienta tecnológica para familias y cuidadores, conocida de ahora en adelante como DEAT en la aplicación móvil que a mediano plazo contemplará cuestionarios hasta los cuatro años de edad y materiales audiovisuales de apoyo. Dicho estudio resulta novedoso desde el campo de la estimulación y atención temprana relativamente reciente en Costa Rica.

2 Como referente teórico podemos citar que en el año 2000 se publica en España el "Libro Blanco de Atención Temprana", obra con aportes significativos para el abordaje de los diferentes profesionales y colaboradores en dicho ámbito para la niñez y coincidentemente es hasta este momento que se empieza a distinguir el valor que tiene la detección y atención temprana como pilar fundamental en la prevención de la discapacidad para la infancia que presenta algún riesgo biológico y/o social, que comienza a alcanzar a algunos países latinoamericanos, entre ellos Costa Rica.

En Costa Rica se ha investigado poco con referencia a la estimulación y atención temprana como estrategia de prevención de la discapacidad en niños nacidos bajo condiciones de riesgo biológico. Por ejemplo: en el año 2011 se inicia la investigación titulada "Estudio comparativo acerca de la implementación de programas de 
estimulación temprana para niños prematuros atendidos en el Estancia Infantil № 2 de la ciudad de Puebla (México) y el CINAI de la ciudad de Goicoechea (Costa Rica): una propuesta de atención temprana para la prevención de la discapacidad"

Dicha investigación finaliza en el año 2013, reflejando resultados de mucha importancia, entre ellos se reconoce la relación entre prematuridad, riesgo biológico y discapacidad en el área de salud, pero no está claro el protocolo que se debe llevar a cabo para la atención temprana de las niñas y niños prematuros (Baxter et al., 2013).

Por otra parte, en dicha investigación y publicación del artículo científico se encontró que no hay una adecuada coordinación entre los sectores de salud y educación referente a esta población, pero sí existe una visión clara de lo que se requiere en este proceso. Por eso se plantea la importancia de establecer equipos de trabajo con miembros de ambas áreas para la atención de esta población, que además, deberían orientar y capacitar a las familias para que se involucren en el proceso de estimulación y atención temprana, ésta tarea debe ser considerada como una obligación, no una opción, para la prevención o disminución de una posible alteración del desarrollo en niños con una condición de riesgo como el caso de la prematuridad. Por último, se considera que un protocolo de atención temprana va a facilitar los procesos de inclusión al sistema educativo formal de los niños. (Baxter et al., 2013).

6 Para el análisis estadísticos del proyecto se revisa la bibliografía existente en otras latitudes, entre ellas las tesis de Alcantud (2012) porque la DEAT se inspiró en la propuesta de dicho autor para referencia y guía metodológica para conocer la forma en que se habían realizado los respectivos estudios de validez y confiabilidad para tropicalizar al entorno costarricense, ya que toda medición o instrumento de recolección de datos debe reunir dos requisitos básicos, los cuales son: la confiabilidad del instrumento que se refiere al grado en que su aplicación repetida al mismo sujeto $u$ objeto produce resultados similares o consistentes con mediciones previas. Se pretende demostrar esta confiabilidad en una segunda etapa, que permita la medición en una forma práctica y objetiva y por otro lado la validez que se refiere al grado en que un instrumento de medición realmente mide la variable que pretende medir.

\section{Metodología}

7 La investigación se concibe desde un enfoque mixto, recolecta, analiza y vincula datos cuantitativos y cualitativos para dar respuesta a un problema determinado en forma longitudinal. En un primer momento de esta etapa el enfoque será predominantemente cuantitativo, se requiere obtener y registrar datos, lo que se conoce como datos duros que reflejen la realidad del país en materia de atención temprana y que permitirán impactar a mediano plazo en políticas públicas. Posteriormente, se ahondará en situaciones más individualizados y en la realidad que los envuelve, por lo que se requerirán técnicas como estudios de casos e historias de vida y que recibirán un análisis cualitativo de la información (Hernández, 2010).

Plantea una propuesta investigativa para la validación de una herramienta tecnológica que será utilizada en la detección, estimulación y atención temprana de niños y niñas, nacidos con o sin condición de riesgo biológico, con el fin de prevenir una posible condición de discapacidad, mediante la aplicación de un protocolo en línea, accesible a los familiares y cuidadores de esta población. 
El proceso de construcción de la herramienta tecnológica se inicia en el 2015 con la elaboración de los cuestionarios del desarrollo integral. Posteriormente se realiza una validación de experto; la cual se compone por 5 profesionales extranjeros y 7 colaboradores nacionales. Para el 2016 se elabora la herramienta tecnológica con un registro del encargado, registro del niño, cuestionarios del desarrollo integral de 1 mes a 24 meses y el informe de los datos suministrados por las familias, el cual se brinda de manera cuantitativa. Paralelamente se trabaja en el diseño gráfico de la herramienta, para el cual se plantea portadas que representan las edades de los cuestionarios. Finalmente se inicia el pilotaje de la herramienta tecnológica el cual se detallará a continuación.

\section{Cuadro $N^{\circ} 1$ Proceso de Construcción de DEAT}

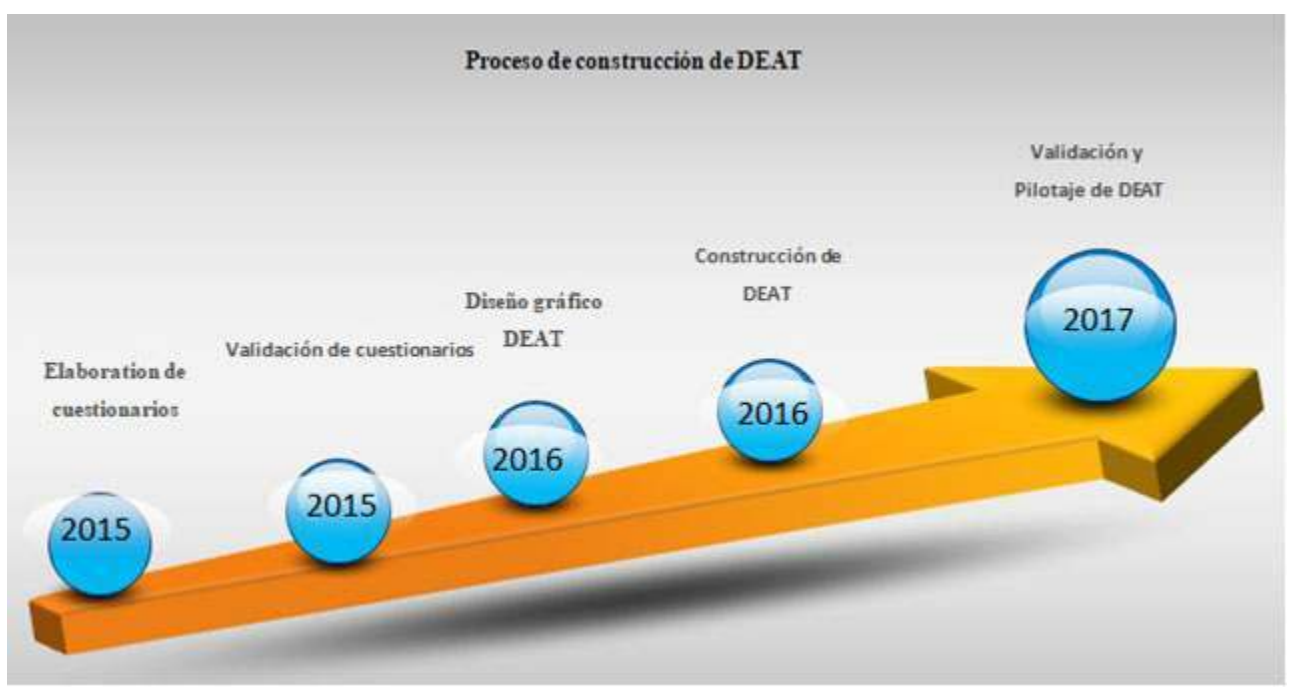

Validación de la Herramienta Tecnológica de estimulación temprana para familias costarricenses. Fuente: Elaboración propia

\section{Aporte Estadístico}

El objetivo en esta investigación desde la visión estadística es determinar si DEAT es fácilmente aplicable y entendible no sólo por los profesionales, sino también por los padres y/o cuidadores. Además de contar con datos científicos que respalden la aplicación de la estimulación y atención temprana como forma de prevenir una condición de discapacidad; por lo que esta investigación es de carácter pionero inclusive a nivel Centroamericano. Es por la relevancia del proyecto y por tener cobertura nacional para impactar en políticas públicas a mediano y largo plazo que este debe incluir el aporte estadístico para la correcta medición de la atención temprana.

11 En primer lugar para la validez del cuestionario o formulario se debe de aplicar la herramienta a un número de niños suficientemente amplio para que las pruebas estadísticas a utilizar tengan consistencia, eso sí tomando en cuenta que no se trata de un muestreo puesto que inicialmente es una prueba de cuestionario para validar la herramienta.

Dado que no conocemos el $\mathrm{N}$ de la población, estimamos que el tamaño muestral (error $=.069$ y un nivel de confianza $\alpha=0.05$ y para el valor máximo de varianza 
poblacional $\mathrm{pq}=0,25)$ es de 200 familias a nivel global, reiterando que el objetivo de la prueba del cuestionario o formulario de la herramienta no es un estudio poblacional, ni un estudio epidemiológico, es más bien una prueba de la herramienta, pero hemos creído oportuno presentar los cálculos del error muestral.

Específicamente para la medición de los niños con o sin riesgo biológico, se utiliza la herramienta por medio de las escalas del desarrollo, que son instrumentos que permiten una adecuada exploración del nivel de crecimiento infantil en las áreas del desarrollo integral: motriz, audición, comunicación y lenguaje; socio emocional y cognitiva (Alcantud, 2012). Escalas que inicialmente serán revisadas por el equipo investigador, posteriormente se van a seleccionar diferentes estratos o segmentos de edades para la aplicación de los instrumentos ó pilotaje, con lo que se trabajará la validez y confiabilidad por último el estudio longitudinal propone determinar el efecto en el desarrollo de los niños.

El proceso que se lleva a cabo para la construcción de la herramienta "Detección, estimulación y atención temprana para la prevención de la discapacidad en niños y niñas costarricenses con una condición de riesgo biológico herramienta tecnológica para familias y cuidadores", incluye la revisión bibliográfica, el análisis de los reactivos o ítems utilizados para cada segmento de edad de las tesis de Alcantud (2012), el estudio de la validez y confiabilidad de los reactivos, la selección de los informantes y la prueba piloto. La valoración del desarrollo adquiere importancia paso previo para la implementación de planes de estimulación en las áreas en que se presente un bajo desempeño (Garrido y Madriz, 2015)

\section{Lista de reactivos o ítems}

El equipo investigador en conjunto construirá, contextualizará y validará las escalas del desarrollo de los niños con riesgo biológico que conformarán la herramienta tecnológica para familias y cuidadores, a partir de la descripción y listados presentados en las tesis de Alcantud (2012). Ante la falta de datos estadísticos sobre el porcentaje de niños que presentan las conductas, se optó por realizar dos tipos de análisis.

En primer lugar, se realizó un análisis cualitativo de los ítems, rehaciendo la redacción en unos casos, y en otros, una reclasificación en áreas diferentes, e incluso en algún caso en niveles de edad diferente. Una vez producido este primer filtrado de ítems (eliminación de duplicaciones, reclasificación, mejora de la redacción, entre otros), se procedió a la evaluación por expertos.

Posteriormente, cuando se cuente con la información arrojada por el pilotaje de la herramienta, se realizará el respectivo análisis psicométrico, para determinar si en la escala existen ítems que deben ser eliminados para la correcta medición del riesgo biológico, como se detalla a continuación.

Por la forma en que se construye la herramienta tecnológica los datos obtenidos permiten no solo la interpretación y acercamiento al objeto de estudio, sino también ir aplicando la confiabilidad a la misma herramienta. 


\section{Plan piloto}

19 En un inicio se planteó elaborar el plan piloto en la provincia de Alajuela, cantón San Ramón con el propósito probar el instrumento que recopilará información de los niños y niñas en edades de un mes a veinticuatro meses. Durante el proceso de investigación del proyecto, el equipo de investigadores de la Universidad Estatal a Distancia (UNED) ha trabajado conjuntamente con personeros de la Caja Costarricense de Seguro Social (CCSS), Se implementa un Sistema de Atención Integral e Intersectorial del Desarrollo de la Niñez (SAIID). Por ello, se estableció como premisa trabajar en los Ebais de diversos distritos de la comunidad de San Ramón. Sin embargo se presentó una limitante, la cual fue que al solicitar la aprobación de los directores de los centros de salud, se hace la aclaración de que para todos los proyectos de investigación, los investigadores deben tener el curso de buenas prácticas clínicas. Ningún colaborador de la CCSS tiene este curso, solo la coordinadora del proyecto de la UNED.

20 Se pretende ajustar e implementar un instrumento desarrollado en las tesis de (Alcantud,2012), para así, captar la información de los menores en las edades: 0-6 meses, 9 meses, 12 meses, 15 meses, 18 meses y 24 meses, para determinar el riesgo biológico y categorizar, a fin de implementar diversas técnicas que apoyarán su desarrollo y bienestar.

21 El instrumento se va a aplicar a niños a lo largo y ancho del país, por ello, es necesario en primera instancia determinar si el cuestionario desarrollado en la tesis de Alcantud y con base a las modificaciones y tropicalización desarrolladas por el equipo multidisciplinario de la UNED a cargo de la investigación en Costa Rica, permite la mejor captación de la información que será insumo para el desarrollo de la investigación y que posteriormente, se utilizará para desarrollar análisis descriptivo, el establecimiento de perfiles y análisis de tendencia y de seguimiento que son empleadas en el argot estadístico.

22 Además, como las escalas fueron aplicadas y validadas en otras latitudes, si no son expuestas al pilotaje para su correspondiente validación en el contexto nacional podrían arrojar resultados equivocados en las mediciones adecuadas del riesgo biológico, puesto que se podrían introducir ítems que perjudiquen la medición de dicha escala.

23 Hay que considerar que lo importante en este punto es la prueba del cuestionario e identificar si el encargado del menor comprende claramente las instrucciones y cuestionamientos que se le brindan. Por ende, en la investigación desarrollada en Alcantud se previó la aplicación de una prueba piloto que brindó luces sobre la facilidad y comprensión del instrumento.

24 Este tipo de ejercicio es común a la hora de evaluar instrumentos, por ejemplo, en el Censo 2011- CR se utilizó este tipo de enfoque para evaluar si el cuestionario desarrollado era comprensible y que las preguntas desarrolladas permitirán capturar la información idóneamente y con la confiabilidad necesaria. Es por ello que el instrumento del Censo 2011 se probó en el cantón de Palmares, zona que cumple con requisitos socioeconómicos para su evaluación. 


\section{Selección de los informantes}

En un primer abordaje para la implementación del cuestionario de la investigación DEAT se contempló algunos centros de atención primaria y secundaria de la Caja Costarricense de Seguro Social ya que esta institución es contraparte esencial para el desarrollo del proyecto ; específicamente, un factor clave que brinda la Institución son estadísticas de los nacimientos en el país y sobre todo de los niños nacidos bajo condición de riesgo biológico población meta del proyecto ; es por esto que se solicitó a las compañeras investigadoras y parte del proyecto la posibilidad de contar con los establecimientos de salud, que se organizan en niveles según la complejidad de los pacientes y procedimientos que se aplican; indicando esta que el área de salud es el primer nivel y el hospital es segundo nivel.

6 En su momento se estableció implementar el instrumento en el cantón de San Ramón debido a que cuenta con un total de 13 distritos y en los cuales ubica 18 Ebais y para el 2016, este cantón registra el 9,2\% de la población total de la provincia de Alajuela. Según datos del Censo 2011 hubo un total de 6.307 menores de edades entre los 0 y 4 años y se prevé, con base en las proyecciones poblacionales del INEC, para el 2016 un total de 6.418 menores en ese grupo etario. Además, el cantón en el 2013 presentó un total de 1.206 nacimientos y en el 2014 nacieron 1.246 menores, partos que en su mayoría fueron en el hospital Carlos Luis Valverde Vega (87 \%). La presencia de centros de atención primarios y secundarios en el cantón de San Ramón lo cual permitía dar seguimiento a los menores en las edades señaladas que residen en zonas con características urbanas y rurales.

La CCSS desarrolla diversos programas como: Clínicas de lactancia materna y desarrollo, Sistema de Atención Integral e Intersectorial del Desarrollo de la Niñez mantiene una relación estrecha con la población de interés; esto permitirá identificar prácticamente de forma inmediata el nacimiento de un niño o niña con una condición de riesgo biológico y por medio del carnet de atención integral del niño (antiguo carnet de vacunas) identificarlo por medio de un código asignado por el personal de salud que atendió el parto y con el cual la familia o cuidadores lo ingresaran a la base de datos de la herramienta tecnológica cada vez que accedan a ella para hacer la respectiva valoración periódica, y el correspondiente plan de estimulación y atención temprana.

La presencia de Centros Universitarios en las distintas provincias del país permite dar seguimiento a los menores en las edades señaladas que residen en zonas con características socioeconómicas diversas las diferentes zonas del país. La UNED es una institución de Educación Pública, la única institución de educación superior a distancia, que ofrece carreras universitarias certificadas por el Sistema Nacional de Acreditación de la Educación Superior (SINAES), brinda la oportunidad de cursar carreras técnicas, así como cursos libres en áreas como educación, comunicación y tecnología, idiomas, desarrollo gerencial, entre otras. Estas y otras particularidades convierten los recursos del centro universitario en idóneos para el pilotaje de los cuestionarios. 
Cuadro $\mathrm{N}^{\circ}$ 2: Distribución de los Centros Universitarios, Universidad Estatal a Distancia

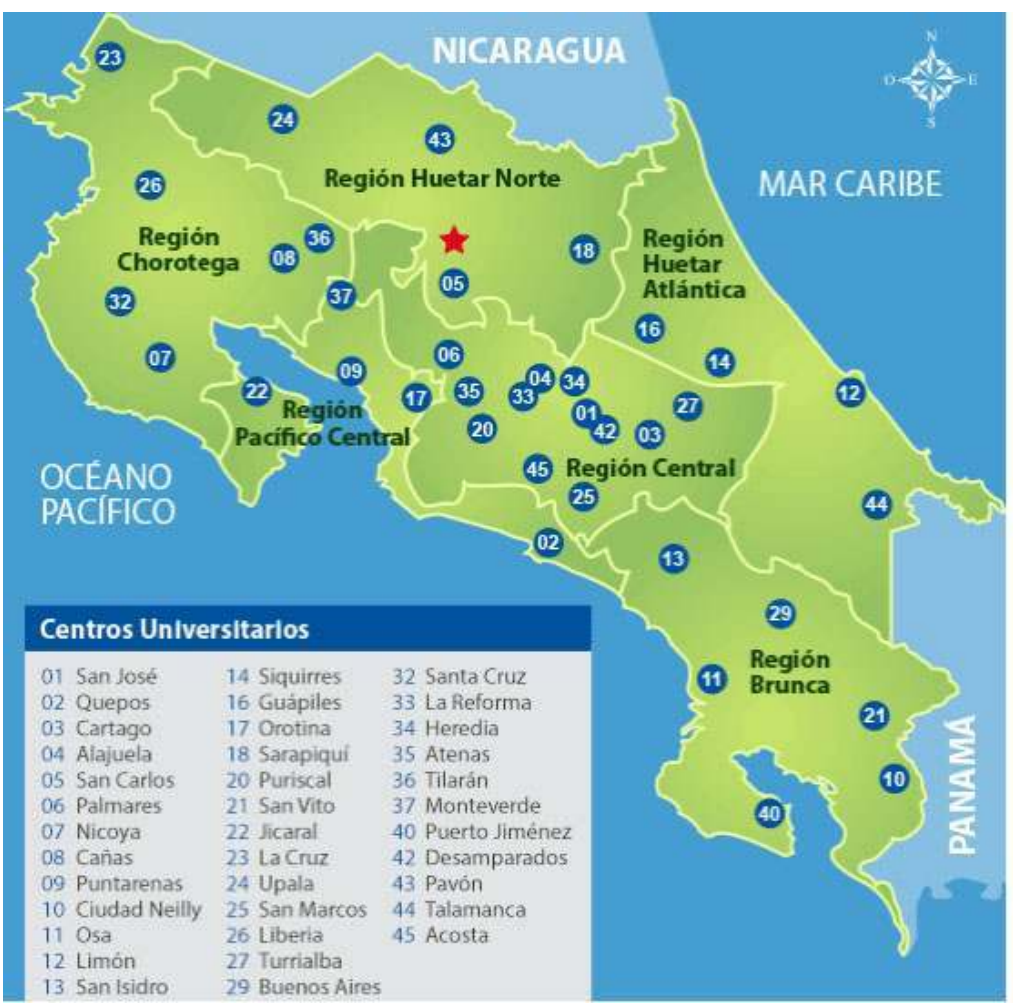

Fuente: Sitio web http://www.uned.ac.cr/index.php/centros-universitarios

La UNED desarrolla diversos programas y mantiene una relación estrecha con la población de interés; esto permitirá identificar niños o niñas con una condición de riesgo biológico. Por ello, el pilotaje conlleva la aplicación de 150 instrumentos que permitirán evaluar el cuestionario, este número se sustenta en lo desarrollado en la tesis de Alcantud y diversos escenarios desarrollados, los cuales valoran las limitaciones señaladas con anterioridad.

Cuadro $N^{\circ} 3$ Costa Rica: Distribución de los instrumentos a aplicar según provincia y Centro de Estudio Universitario (CEU). 2016

\begin{tabular}{lcl}
\hline \multicolumn{1}{c}{ Provincia } & $\begin{array}{c}\text { Distribución de } \\
\text { Instrumentos }\end{array}$ & \multicolumn{1}{c}{ CEU Elegidos } \\
\hline San José & 20 & San José y Desamparados \\
Heredia & 20 & Heredia \\
Cartago & 20 & Cartago \\
Alajuela & 20 & Alajuela \\
Limón & 24 & Limón y Ciudad Neilly \\
Puntarenas & 22 & Puntarenas \\
Guanacaste & 24 & Liberia y Monte Verde \\
\hline
\end{tabular}

Fuente: Elaboración propia, con base en la información de los Centros Universitarios. 2017 


\section{Resultados}

te

Los cuestionarios son sistémicos, respetando las características del desarrollo integral de cada edad y llevando una secuencia donde las características previas de cada etapa son base para iniciar la etapa siguiente.

En una primera etapa los cuestionarios del desarrollo que llevará la herramienta tecnológica, se distribuyen tomando en cuenta la edad cronológica de los niños y las áreas del desarrollo motriz, audición, comunicación y lenguaje, socio-emocional y cognitiva.

Cuadro $n^{\circ} 4$ : Escala del Desarrollo

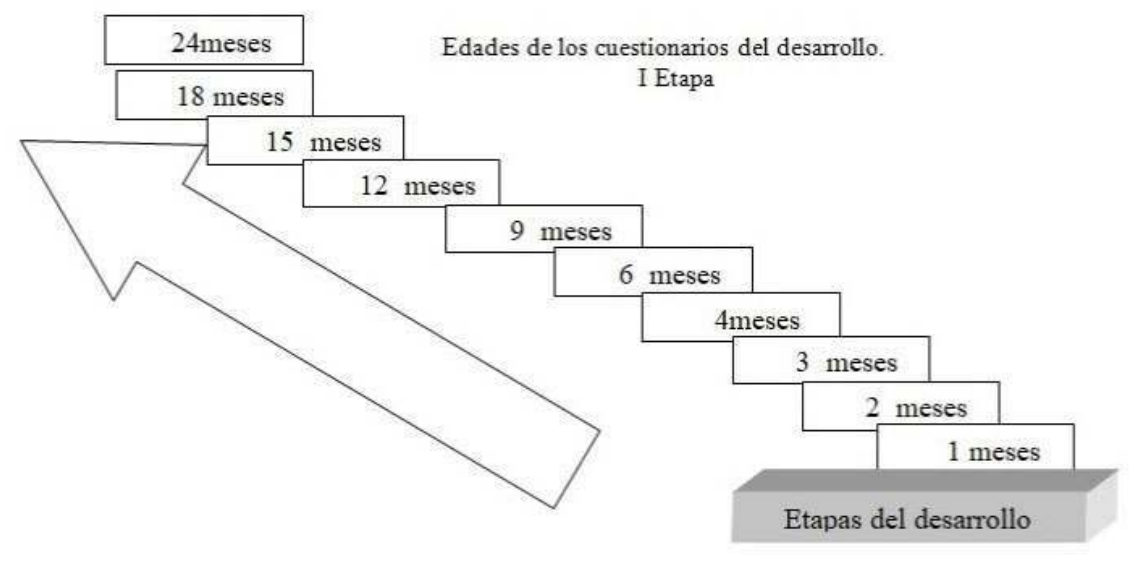

FUENTE: Elaboración propia, con base en la información de los Centros Universitarios. 2017.

Al inicio de cada etapa, el usuario de DEAT encontrará información general de características del desarrollo, esperadas para cada edad. Posteriormente se presenta signos de alerta que tiene como objetivo, avisar a los padres de familias y/o cuidadores que presten mayor atención al desarrollo integral de los niños a su cuidado. Los cuestionarios del desarrollo permitirán a los familiares y/o cuidadores del menor, registrar las destrezas del desarrollo correspondiente a la edad cronológica del niño. El producto final de este apartado del proyecto son diez cuestionarios del desarrollo integral del menor, que van desde el primer mes de desarrollo hasta los 24 meses. Finalizado el proceso de registro de las destrezas se le brindará un informe general sobre el desarrollo del infante. Además de la lista de diferentes instituciones a nivel nacional para buscar mayor información y orientación de ser necesario.

Al respecto todos los cuestionarios más sus respectivos audiovisuales de evaluación así como de planes de atención temprana se encuentran integrados en una herramienta tecnológica desarrollada por un ingeniero en sistemas genera después de la inscripción de los participantes, recordatorios automáticos a las familias de cada cuanto deben ingresar, también emite un reporte de resultado del cuestionario (formato PDF). Desarrollo web: Gregory Hidalgo Ramírez con las siguientes características:

Front end

\begin{tabular}{|l|l|l|}
\hline NOMBRE & VERSIÓN & FUNCIÓN \\
\hline
\end{tabular}




\begin{tabular}{|l|l|l|}
\hline HTML & 5 & Documento HTML (etiquetas). Se implementó la semántica. \\
\hline Javascript & - & Trabajar con formularios \\
\hline jQuery & 1.12 .1 & Framework de Javascript. \\
\hline CSS & 3 & Lenguaje de programación. \\
\hline Boostrap & 3 & Framework de CSS \\
\hline
\end{tabular}

Back end

\begin{tabular}{|l|l|l|}
\hline NOMBRE & VERSIÓN & FUNCIÓN \\
\hline UBUNTU & 4.14 & Sistema operativo del servidor. \\
\hline APACHE & 5.5 & Servidor \\
\hline PHP & & Lenguaje de programación. \\
\hline PHP Mailer & & \\
\hline PHPEXcel & 1.8 & \\
\hline TCPDF & & Crear reportes en documentos PDF. \\
\hline
\end{tabular}

Bases de datos

\begin{tabular}{|l|l|l|}
\hline NOMBRE & VERSIÓN & FUNCIÓN \\
\hline MYSQL & 5.6 & Guardar la información del proyecto. \\
\hline
\end{tabular}

Herramientas de desarrollo

\begin{tabular}{|l|l|l|}
\hline NOMBRE & VERSIÓN & FUNCIÓN \\
\hline NETBEANS & 8.1 & \\
\hline Sublime Text & 2 & \\
\hline MySQL Workbench & 6.3 & \\
\hline
\end{tabular}

Herramientas de diseño web

\begin{tabular}{|l|l|l}
\hline NOMBRE & VERSIÓN & FUNCIÓN \\
\hline
\end{tabular}




\begin{tabular}{|l|l|l|}
\hline Adobe Ilustrador & - & Propuesta y diseño final del sitio. \\
\hline
\end{tabular}

Herramientas de video

\begin{tabular}{|l|l|l|}
\hline NOMBRE & VERSIÓN & FUNCIÓN \\
\hline Adobe Premiere & - & Edición de los videos para los cuestionaros. \\
\hline
\end{tabular}

Otras herramientas

\begin{tabular}{|l|l|l|}
\hline NOMBRE & VERSIÓN & FUNCIÓN \\
\hline Adobe Experience & - & Crear prototipo antes comenzar el desarrollo. \\
\hline
\end{tabular}

\section{Otros detalles}

Responsive Web Design: Se utiliza diseño responsivo para que DEAT pueda ejecutarse y visualizarse en móviles, tablets, laptops y computadoras de Escritorio.

MVC: Patrón de Diseño Modelo, Vista, Controlador.

Es decir de forma automatizada el sistema DEAT, desarrollado en una tecnología móvil compatible con celulares, tabletas o computadoras provee al usuario un reporte en formato PDF cuando finaliza con éxito el cuestionario con datos cuantitativos que le pueden orientar en la toma de decisiones y sobretodo en una derivación temprana al presentarse para un análisis más detallado a un profesional en saludo por medio de la alianza estratégica con la ccss.

\section{Discusión de resultados}

En una realidad, no ajena al contexto social y económico costarricense, donde el desarrollo integral de los menores es atendido hasta que se incorporan en los sistemas formales de educación. Es evidente la necesidad de que, ante factores de riesgo biológico asociados a una condición como la prematuridad, se hace indispensable una intervención temprana e implementar programas y servicios que puedan dar respuesta a esta población en el entorno apropiado y con el trabajo conjunto de las diferentes instancias, profesionales correspondientes y de las familias o cuidadores.

En la presentación de la Herramienta (DEAT) a las familias, han mostrado gran interés por el tema de atención temprana. La asistencia a la charla y demostración de materiales de estimulación y exploración de la herramienta ha sido satisfactoria, superando el número de participantes que se tenía previsto. La provincia de Heredia fue la única donde la participación fue escasa ; solamente dos personas se presentaron a la charla.

37 Con respecto a la herramienta tecnología las familias han manifestado que el uso es sencillo, fácil de aportar y acceder a la información. 
38 Al referirse a los cuestionarios nos indican que en algunos ítems requieren una ayuda adicional para entender lo que se les está solicitando. Ante la pregunta que si con una ayuda audiovisual, la información se comprendería más fácilmente la respuesta general es que sí.

Solicitan que algunos de los ítems de las destrezas deben de llevar un ejemplo de lo que se pide que observe ; para lo cual el apoyo del video sería de mucha utilidad

A continuación se presenta los lugares donde se ha realizado la validación DEAT y los cuestionarios del desarrollo.

Cuadro $N^{\circ}$ 5: Distribución de Centros Universitarios.Validación de DEAT

\begin{tabular}{|l|l|}
\hline Fecha & Lugar \\
\hline 1-6-2017 & Centro Universitario San José \\
\hline 3-6-2017 & Centro Universitario Limón \\
\hline 9-6-2017 & Centro Universitario Puntarenas \\
\hline 14-6-2017 & Centro Universitario Heredia \\
\hline 15-6-2017 & Centro Universitario Cartago \\
\hline
\end{tabular}

Fuente: Elaboración propia

41 Al realizar la revisión de los ítems contenidos en cada uno de los cuestionarios, se logró identificar al menos un ítem que estaba escrito en sentido negativo, lo cual puede crear tanto confusión en la persona que llena la herramienta como la persona que la procesa, ya que los ítems deben estar redactados en forma positiva, ya que de lo contrario generan una contradicción lógica por lo que se procedió a realizar el cambio del sentido de la pregunta y así eliminar el problema.

$42 \mathrm{Al}$ obtener los datos del pilotaje se realizarán los análisis estadísticos respectivos para la validación de las escalas, con el propósito de identificar si existen ítems que deben ser eliminados o mantenidos en dichas escalas.

El diseño DEAT permitirá generar una base de datos con una serie de indicadores que serán sujetos de un análisis cuantitativo y se realizará una selección de algunos de los participantes para trabajar con ellos estudios de caso e historias de vida para el análisis cualitativo.

El análisis cuantitativo (I fase) se hará de forma digital utilizando diferentes software para procesar y analizar los datos cuantitativos que se obtendrán por medio de la base de datos que contempla la herramienta tecnológica.

Por un compromiso con la niñez costarricense y las familias es que se le brindará la oportunidad de que sean partícipes activos del desarrollo y crecimiento de sus hijos. La atención temprana forma parte de un reto, es responsabilidad de toda la sociedad. 


\section{BIBLIOGRAFÍA}

Francisco-Alcantud, Alonso- Rico, Sistema de detección precoz de los trastornos del desarrollo. Respuestas flexibles en contextos educativos diversos, Consejería De Educación, Formación y Empleo, Murcia, 2012.

Jorge Baxter, Linda Madriz, Lorena Mora, Prematuridad y estimulación temprana: ¿un binomio determinante para la prevención de la discapacidad ?, Universidad Estatal a Distancia, Mercedes de Montes de Oca, 2012.

Jorge Baxter, Linda Madriz, Lorena Mora, Prematuridad y estimulación temprana: ¿un binomio determinante para la prevención de la discapacidad, Universidad Estatal a Distancia, Mercedes de Montes de Oca, 2012.

John, Buteler, Judith D Singer, JudithS Palfrey, Deborah K Walker, "Health insurance coverage and physician use among children with disabilities: findings from probability samples in five metropolitan areas", Pediatrics, 79(1), 1987, p. 89-98.

Costa Rica. Instituto Nacional de Estadística y Censos. (2012). X Censo Nacional de Población y VI de Vivienda, 2011.

Libro Blanco de la Atención Temprana, Real Patronato sobre Discapacidad, Madrid, [en línea] http:// www.infodisclm.com/libroblancoat.htm. Consultado el 8 de mayo 2017.

Frances P Glascoe, Shull Marcias, "How you can implement the AAP's new policy on developmental and behavioral screening. (Developmental Screening)," Contemporary Pediatrics, 2003.

Miguel Gómez, Elementos de estadística descriptiva, Universidad Estatal a Distancia, Mercedes de Montes de Oca, 1998.

LaszloKish, Muestreo de Encuestas, Trillas, México, 1972.

Judith S Palfrey, Judith D Singer, Deborah K Walker, John A Butler, "Early identification of children's special needs: a study in five metropolitan communities" The Journal of pediatrics, 111(5), 1987, p. 651-659.

Roberto Hernández, Carlos Fernández, Pilar Baptista, Metodología de la investigación, Editorial McGrawHill. Estado de México, 2010.

\section{RESÚMENES}

Se presenta el proceso de validación y pilotaje de los instrumentos del proyecto "Detección, estimulación y atención temprana para la prevención de la discapacidad en niños y niñas costarricenses con una condición de riesgo biológico: Herramienta Tecnológica para familias y cuidadores". El objetivo principal de dicha herramienta es que pueda ser utilizada por familias, cuidadores y profesionales. Tiene dos componentes básicos, el primero está integrado por una serie de escalas de desarrollo, que comprenden desde el 1 mes de vida hasta los 48 meses de vida. El segundo, tiene un enfoque formativo para dotar de conocimientos y estrategias para la estimulación y atención temprana de los destinatarios por medio de una serie de materiales audiovisuales. La principal ventaja es que al ser parte de la tecnología móvil es asincrónico y esta disponibilidad facilita reducir las barreras de índole cultural, económicas y geográficas. 
On présente le processus de validation et de pilotage des instruments du projet "Détection, stimulation et attention précoce pour la prévention des handicaps chez les enfants costaricains qui présent un risque biologique: outil technologique pour les familles et les aidants". L'objectif principal de cet outil est qu'il peut être utilisé par les familles, les soignants et les professionnels. Il a deux composants de base, le premier est intégré par une série d'échelles de développement, allant de 1 mois de vie à 48 mois de vie. La seconde a une approche formative pour fournir des connaissances et des stratégies pour stimuler et attirer l'attention des destinataires à travers une série de documents audiovisuels. Le principal avantage est qu'en faire partie de la technologie mobile il est asynchrone et cette disponibilité facilite la réduction des obstacles culturels, économiques et géographiques.

The validation process and pilot plan is presented for the project "detection, early stimulation and care for the prevention of disability in Costa Rican children within a biological risk condition: Web Protocol for families and caregivers." The main objective is to design a tool that can be used by professionals, caregivers and families. Its use will contribute to the prevention, detection, stimulation and intervention of children in a biological risk condition.

The tool will have two components; one will provide a protocol with scales of integral development that goes from 1 to 48 months. The other component is a training approach that will provide knowledge and strategies for early stimulation and care recipients.

The main advantage of the Web tool is that is going to be available for smart phones technology, which will reduce the cultural, economic and geographical barriers.

\section{ÍNDICE}

Palabras claves: estimulación temprana, atención temprana, prematuridad, condición de riesgo biológico y/o social, neuroplasticidad

Keywords: early stimulation, early warning, prematurity, bioligical risk condition, neuroplasticity

Mots-clés: stimulation précoce, soins précoces, prématurité, état du risque biologique et / ou social, neuroplasticité

\section{AUTORES}

\section{LINDA MADRIZ}

Universidad Estatal a Distancia, Costa Rica

\section{LORENA SOTO}

Universidad Estatal a Distancia, Costa Rica

\section{LENNIN COLLADO}

Universidad Estatal a Distancia, Costa Rica

\section{ALEX SEGURA}

Universidad Estatal a Distancia, Costa Rica 